\title{
Matrix model of inhomogeneous medium with circular birefringence in single scattering case
}

\author{
S.N. Savenkov, Ye.A. Oberemok, V.V. Yakubchak, O.I. Barchuk \\ Taras Shevchenko Kyiv National University, Radiophysics Department, \\ 64, Volodymyrska str., 01601 Kyiv, Ukraine, \\ Phone: (380-44)526-05-80; e-mail: sns@univ.kiev.ua
}

\begin{abstract}
The paper is devoted to the analysis of light scattering by inhomogeneous circular birefringent media in a single scattering case. The object under investigation is a circular birefringent crystalline slab with surface inhomogeneity. For the analysis, we derive the Mueller matrix model for this media and use Cloude's coherency matrix method. Sample calculations are given for quartz $\mathrm{SiO}_{2}$ and paratellurite $\mathrm{TeO}_{2}$.
\end{abstract}

Keywords: light scattering, depolarization, Mueller matrix, circular birefringence, Cloude's coherency matrix, entropy.

Manuscript received 04.03.09; accepted for publication 18.03.09; published online 20.03.09.

\section{Introduction}

Interaction of medium with a beam of polarized light can be described as transformation of the input Stokes parameters into output ones by the Mueller (scattering) matrix that contains information related to the anisotropic and depolarization properties of the medium. Consequently, a detailed interpretation of the Mueller matrix elements may be used to characterize the physical properties of studied medium [2-4].

In spite of the fact that typically the circular birefringence effect is about $10^{3}-10^{5}$ times smaller than that for linear birefringence, polarization of radiation is affected much more than that expected for this small value. The Mueller matrix of circular birefringence is [4]:

$$
\mathbf{M}^{C B}(\varphi)=\left(\begin{array}{cccc}
1 & 0 & 0 & 0 \\
0 & \cos (2 \varphi) & \sin (2 \varphi) & 0 \\
0 & -\sin (2 \varphi) & \cos (2 \varphi) & 0 \\
0 & 0 & 0 & 1
\end{array}\right),
$$

where $\varphi$ is the value of circular birefringence.

In this paper, we derive the Mueller matrix of the circular birefringent crystalline slab with surface inhomogeneity and analyze information on medium anisotropy and depolarization properties containing in the elements of derived matrix using an additive model basing on the so-called Cloude coherency matrix $[5,6]$.

\section{Mueller matrix model}

As shown earlier [1], the Mueller matrix of elliptically birefringent crystalline slab with surface inhomogeneity (roughness) in the eigen coordinate system has the following form:

$$
\mathbf{M}^{\text {eigen }}=\left(\begin{array}{cccc}
1 & \frac{\Phi_{11}-\Phi_{22}}{\Phi_{11}+\Phi_{22}} & 0 & 0 \\
\frac{\Phi_{11}-\Phi_{22}}{\Phi_{11}+\Phi_{22}} & 1 & 0 & 0 \\
0 & 0 & \frac{\Phi_{12}+\Phi_{21}}{\Phi_{11}+\Phi_{22}} & i \frac{\Phi_{12}-\Phi_{21}}{\Phi_{11}+\Phi_{22}} \\
0 & 0 & -i \frac{\Phi_{12}-\Phi_{21}}{\Phi_{11}+\Phi_{22}} & \frac{\Phi_{12}+\Phi_{21}}{\Phi_{11}+\Phi_{22}}
\end{array}\right),
$$

where

$$
\begin{aligned}
& \Phi_{u v}=\Phi_{u v}^{b r f}\left(\left(1-\eta_{u v}\right) \exp \left\{-\left(\frac{k \rho w}{2 z}\right)^{2}\right\}+\right. \\
& \left.+1 \frac{\eta_{u v}}{\sigma_{u v}^{2} w^{2}+1} \exp \left\{-\frac{1}{\sigma_{u v}^{2} w^{2}+1}\left(\frac{k \rho w}{2 z}\right)^{2}\right\}\right) \\
& \Phi_{u v}^{b r f}=\exp \left(i k\left(n_{u}-n_{v}\right) \bar{h}-k^{2} \sigma_{h}^{2}\left(n_{u}-n_{v}\right)^{2} / 2\right) \\
& w=d\left(1+4 z^{2} / d^{4} k^{2}\right)^{1 / 2} \\
& \sigma_{u v}^{2}=k^{2} \sigma_{h}^{2}\left(n_{u}-1\right)\left(n_{v}-1\right)
\end{aligned}
$$


$\eta_{u v}=1-\exp \left(-\sigma_{u v}^{2}\right)$

$k=2 \pi / \lambda$ is the wave number; $\bar{h}$ is the thickness of a crystalline slab; $n_{u}$ and $n_{v}$ denotes refractive indexes of a medium associated with its elliptical eigenpolarizations, and $d$ is the beam radius.

If to assume that the Mueller matrix Eq. (2) is presented in circular eigen coordinate system, i.e. $n_{u}=n_{r}$ and $n_{v}=n_{l}$ denotes in this case the refractive indexes associated with circular, right and left, eigenpolarizations, then this matrix corresponds to a matrix model of the medium characterized by circular birefringence at least in a given direction of light propagation.

If the Mueller matrix in eigencoordinate system, $\mathbf{M}^{\text {eigen }}$, Eq. (2) is known, then the Mueller matrix in the laboratory system, $\mathbf{M}^{l a b}$, can be found using a well known algebra [7] with the corresponding transformation matrix associated with circular eigenvectors:

$$
\mathbf{E}_{e 1, e 2}=\left(\begin{array}{c}
1 \\
\pm i
\end{array}\right) \text {. }
$$

Then, the Mueller matrix in the laboratory coordinate system takes the form:

$$
\mathbf{M}^{l a b}=\left(\begin{array}{cccc}
1 & 0 & 0 & \frac{\Phi_{11}-\Phi_{22}}{\Phi_{11}+\Phi_{22}} \\
0 & \frac{\Phi_{12}+\Phi_{21}}{\Phi_{11}+\Phi_{22}} & i \frac{\Phi_{12}-\Phi_{21}}{\Phi_{11}+\Phi_{22}} & 0 \\
0 & -i \frac{\Phi_{12}-\Phi_{21}}{\Phi_{11}+\Phi_{22}} & \frac{\Phi_{12}+\Phi_{21}}{\Phi_{11}+\Phi_{22}} & 0 \\
\frac{\Phi_{11}-\Phi_{22}}{\Phi_{11}+\Phi_{22}} & 0 & 0 & 1
\end{array}\right),
$$

\section{Analysis of the derived matrix model}

Next, we examine our model with the widely used crystals: quartz $\mathrm{SiO}_{2}$ and paratellurite $\mathrm{TeO}_{2}$ with elliptical eigenpolarizations, in a general case. The analysis is carried out for exact forward scattering in the case when light propagation occurs in the direction where both crystals exhibit only circular birefringence.

Comparing the structures of the Mueller matrices for a slab of circular birefringent medium without inhomogeneity Eq. (1) and for that with inhomogeneity Eq. (4), one can see that, because of elements $m_{14}$ and $m_{41}$ of the matrix Eq. (4) are generally not equal to zero, the presence of inhomogeneity results in occurrence of new polarization and depolarization effects.

\subsection{The case of $\sigma_{h}=0$}

From Eqs. (2) and (4), setting $\sigma_{h}=0$, we get:

$$
\mathbf{M}_{\sigma_{h}=0}^{l a b}=\frac{2}{1+\frac{z^{2} \lambda^{2}}{d^{4} \pi^{2}}}\left(\begin{array}{cccc}
1 & \cos \left(\frac{2 \bar{h} \pi \Delta n}{\lambda}\right) & -\sin \left(\frac{2 \bar{h} \pi \Delta n}{\lambda}\right) & 0 \\
0 & \cos \left(\frac{2 \bar{h} \pi \Delta n}{\lambda}\right) & \cos \left(\frac{2 \bar{h} \pi \Delta n}{\lambda}\right) & 0 \\
0 & 0 & 1
\end{array}\right) .
$$

The Mueller matrix Eq. (5) is a matrix of circular birefringence which is equivalent to the matrix Eq. (1). Note that when one sets $\bar{h}=0$, the matrix Eq. (5) becomes identity.

\subsection{The case of large inhomogeneities}

In [1] it was shown that when inequality

$\sigma_{h}>\frac{\lambda \sqrt{7}}{2 \pi\left|n_{r}-n_{l}\right|}$

is satisfied, then the matrix Eq. (2) contains only four non-zero elements, these elements form $2 \times 2$ principal minor, and is singular. This is the so-called case of large inhomogeneities.

The Mueller matrix in laboratory coordinate system Eq. (4) in this case takes the following form:

$$
\mathbf{M}_{\sigma_{h} \rightarrow \infty}^{l a b}=\left(\begin{array}{cccc}
1 & 0 & 0 & \frac{\Phi_{11}-\Phi_{22}}{\Phi_{11}+\Phi_{22}} \\
0 & 0 & 0 & 0 \\
0 & 0 & 0 & 0 \\
\frac{\Phi_{11}-\Phi_{22}}{\Phi_{11}+\Phi_{22}} & 0 & 0 & 1
\end{array}\right) .
$$

Table presents the values of inhomogeneity $\sigma_{h}$ for which the inequality Eq. (6) is satisfied and the Mueller matrix has the form Eq. (7) for paratellurite $\mathrm{TeO}_{2}$ and quartz $\mathrm{SiO}_{2}$ crystals. Estimations are derived for the wavelength $\lambda=0.63 \mu \mathrm{m}$.

The derived estimations show that at the given wavelength the case of large inhomogeneity is not actually attainable and, thus, in the range of inhomogeneity $\sigma_{h}<d$ the Mueller matrix of inhomogeneous circularly birefringent medium Eq. (2) is not singular.

In a general case, from Eq. (4) for the output intensity we have:

$I^{\text {out }}=s_{1}^{\text {out }}=1+\frac{\Phi_{11}-\Phi_{22}}{\Phi_{11}+\Phi_{22}} \sin \left(2 \varepsilon^{\text {inp }}\right)$.

Table. Estimations of acceptability of approximations Eq. (6) for paratellurite and quarts crystals at the wavelength $\lambda=0.63 \mu \mathrm{m}$.

\begin{tabular}{|c|c|c|c|}
\hline Crystals & $n_{r}$ & $n_{l}$ & $\sigma_{h}, \mathrm{~m}$ \\
\hline $\mathrm{TeO}_{2}$ & 2.2600 & 2.2594 & 0.0004 \\
\hline $\mathrm{SiO}_{2}$ & 1.5427 & 1.5426 & 0.0020 \\
\hline
\end{tabular}


So, the output intensity depends only on the value of ellipticity $\varepsilon^{i n p}$ of input polarization. In this case, maximum and minimum values of the output intensity are

$I_{\max }^{l a b}=\frac{2 \Phi_{11}}{\Phi_{11}+\Phi_{22}}$,

$I_{\min }^{l a b}=\frac{2 \Phi_{22}}{\Phi_{11}+\Phi_{22}}$,

and are achieved at $\varepsilon^{i n p}= \pm \pi / 4$, respectively.

The degree of polarization of output radiation is

$p^{\text {out }}=\frac{\sqrt{\left(s_{2}^{\text {out }}\right)^{2}+\left(s_{3}^{\text {out }}\right)^{2}+\left(s_{4}^{\text {out }}\right)^{2}}}{s_{1}^{\text {out }}}=$
$\frac{\sqrt{\left(\left(\Phi_{11}-\Phi_{22}\right)+\left(\Phi_{11}+\Phi_{22}\right) \sin \left(2 \varepsilon^{\text {inp }}\right)\right)^{2}+4 \Phi_{12} \Phi_{21} \cos ^{2}\left(2 \varepsilon^{\text {inp }}\right)}}{\left(\left(\Phi_{11}+\Phi_{22}\right)+\left(\Phi_{11}-\Phi_{22}\right) \sin \left(2 \varepsilon^{\text {inp }}\right)\right)}$.

Then $p_{\max }^{\min }=1$, i.e. the case when output radiation is completely polarized, is achieved at $\varepsilon^{i n p}=\pi / 4$, and at $\varepsilon^{i n p}=-\pi / 4$ we have $p^{\text {out }}=\frac{\Phi_{11}}{\Phi_{22}}$. The minimum value of degree of polarization is

$p_{\text {min }}^{\text {out }}=\frac{\sqrt{\left(\Phi_{11}-\Phi_{22}\right)^{2}+4 \Phi_{12} \Phi_{21}}}{\Phi_{11}+\Phi_{22}}$,

and is achieved at $\varepsilon=0^{0}$.

Fig. 1 shows the dependences of minimum values of polarization degree for output radiation $p_{\text {min }}^{\text {out }}$ as a function of the inhomogeneity $\sigma_{h}$ and wavelength $\lambda$.

Let us estimate the accessible minimum value of $p_{\text {min }}^{\text {out }}$. This value corresponds to the case of large inhomogeneity $\sigma_{h} \rightarrow \infty$. Then, using the explicit form of the Mueller matrix for this case Eq. (7), we get:

$$
\begin{aligned}
& \left.p_{\min }^{\text {out }}\right|_{\sigma_{h} \rightarrow \infty}=\frac{\left(\frac{1}{\left(n_{r}-1\right)^{2}}-\frac{1}{\left(n_{l}-1\right)^{2}}\right)}{\left(\frac{1}{\left(n_{r}-1\right)^{2}}+\frac{1}{\left(n_{l}-1\right)^{2}}\right)}= \\
& =\frac{\left(n_{r}\right)^{2}-\left(n_{l}\right)^{2}-2\left(n_{r}-n_{l}\right)}{2+\left(n_{r}\right)^{2}+\left(n_{l}\right)^{2}-2\left(n_{r}+n_{l}\right)},
\end{aligned}
$$

Thus, $p_{\min }^{\text {out }}$ is determined only by the values of refractive indexes for this medium at the given wavelength. This means that the value $p_{\text {min }}^{\text {out }}$ cannot be equal to zero, because from Eq. (13) in this case $n_{r}=n_{l}$.

\subsection{Cloude's coherency matrix method}

A detailed insight into the polarization and depolarization effects of Eq. (4) can be obtained from Cloude's coherency matrix method [5, 6]. For simplicity and without losses of generality, we will analyze the matrix Eq. (4) in its eigencoordinate system, i.e., the matrix of the form Eq. (2).

Then, in general case Cloude's coherency matrix corresponding to the Mueller matrix Eq. (2) takes the form:

$\mathbf{T}=\left(\begin{array}{cccc}\frac{1}{2}\left(1+\frac{\Phi_{12}+\Phi_{21}}{\Phi_{11}+\Phi_{22}}\right) & 0 & 0 & \frac{1}{2}\left(\frac{\Phi_{11}-\Phi_{22}}{\Phi_{11}+\Phi_{22}}+\frac{\Phi_{12}-\Phi_{21}}{\Phi_{11}+\Phi_{22}}\right) \\ 0 & 0 & 0 & 0 \\ 0 & 0 & 0 & 0 \\ \frac{1}{2}\left(\frac{\Phi_{11}-\Phi_{22}}{\Phi_{11}+\Phi_{22}}-\frac{\Phi_{12}-\Phi_{21}}{\Phi_{11}+\Phi_{22}}\right) & 0 & 0 & \frac{1}{2}\left(1-\frac{\Phi_{12}+\Phi_{21}}{\Phi_{11}+\Phi_{22}}\right)\end{array}\right)$.

This matrix has the following eigenvalues

$\lambda_{1}=1+\frac{\sqrt{\left(\Phi_{11}-\Phi_{22}\right)^{2}+4 \Phi_{12} \Phi_{21}}}{\Phi_{11}+\Phi_{22}}$,
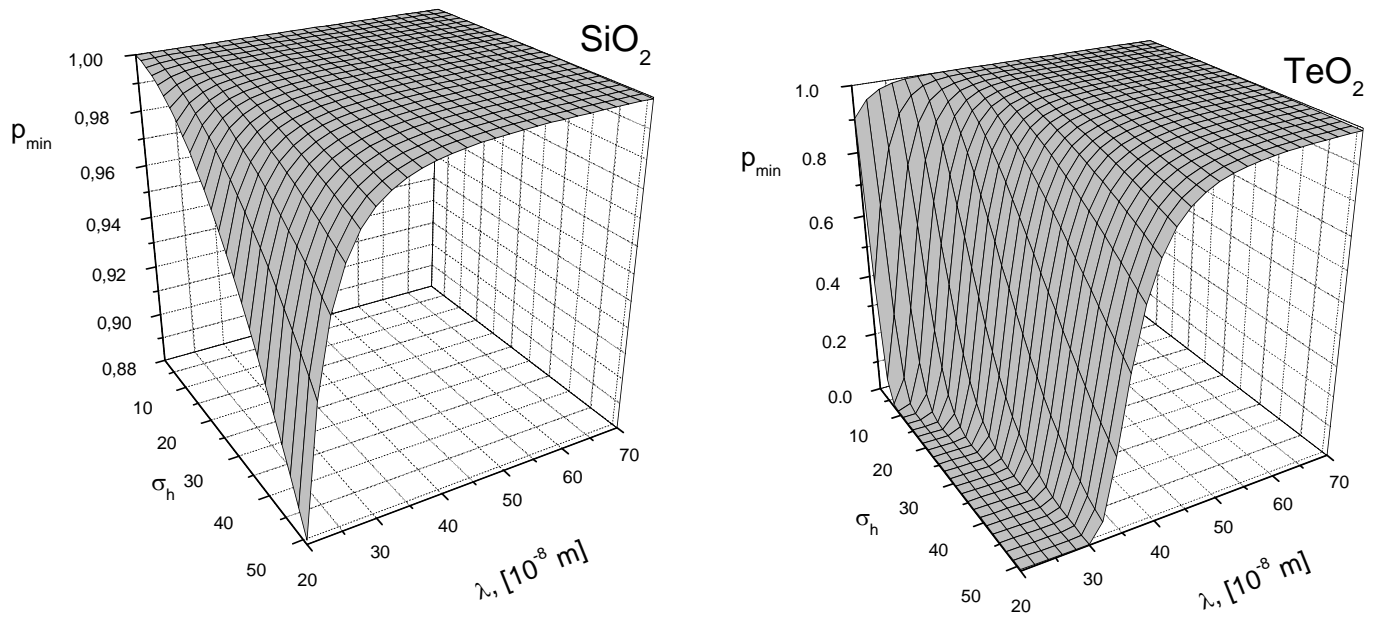

Fig. 1. Dependences of $p_{\mathrm{min}}^{\text {out }}$ on the value of inhomogeneity $\sigma_{h}$ and wavelength $\lambda:$ (a) quartz $\mathrm{SiO}_{2} ;$ (b) paratellurite $\mathrm{TeO}_{2}$. (C) 2009, V. Lashkaryov Institute of Semiconductor Physics, National Academy of Sciences of Ukraine 

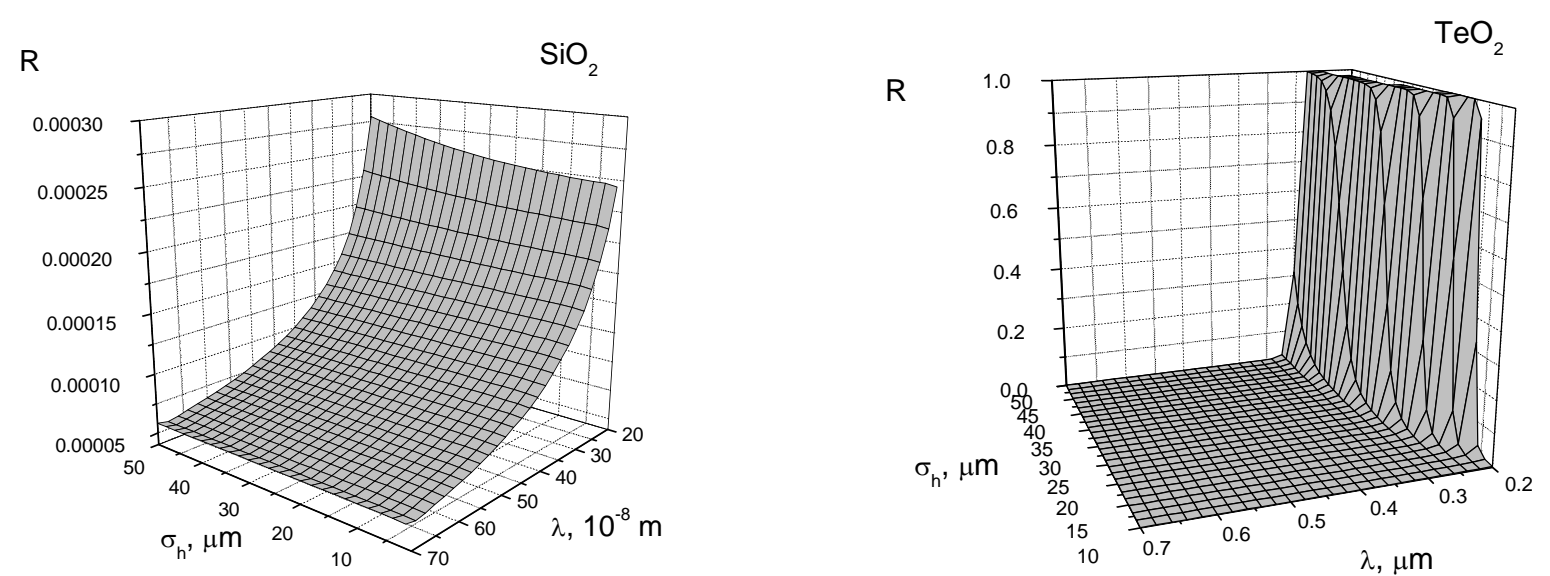

Fig. 2. Dependences of the values of circular dichroism $R$ on inhomogeneity $\sigma_{h}$ and wavelength $\lambda$ : (a) quartz $\mathrm{SiO}_{2} ;$ (b) paratellurite $\mathrm{TeO}_{2}$.

$\lambda_{2}=1-\frac{\sqrt{\left(\Phi_{11}-\Phi_{22}\right)^{2}+4 \Phi_{12} \Phi_{21}}}{\Phi_{11}+\Phi_{22}}$,

$\lambda_{3}=0, \quad \lambda_{4}=0$

and eigenvectors:

$\xi_{1}=\left(\begin{array}{c}\frac{\Phi_{12}+\Phi_{21}+\sqrt{\left(\Phi_{11}-\Phi_{22}\right)^{2}+4 \Phi_{12} \Phi_{21}}}{\Phi_{11}-\Phi_{22}-\Phi_{12}+\Phi_{21}} \\ 0 \\ 0 \\ 1\end{array}\right)$,
$\xi_{2}=\left(\begin{array}{c}\frac{\Phi_{12}+\Phi_{21}-\sqrt{\left(\Phi_{11}-\Phi_{22}\right)^{2}+4 \Phi_{12} \Phi_{21}}}{\Phi_{11}-\Phi_{22}-\Phi_{12}+\Phi_{21}} \\ 0 \\ 0 \\ 1\end{array}\right)$,

$\xi_{3}=\left(\begin{array}{l}0 \\ 0 \\ 1 \\ 0\end{array}\right), \quad \xi_{4}=\left(\begin{array}{l}0 \\ 1 \\ 0 \\ 0\end{array}\right)$.

The Jones matrices associated with eigenvalues $\xi_{1}$ and $\xi_{2}$ Eqs. (18) and (19), which correspond to two non-zero eigenvalues $\lambda_{1}$ and $\lambda_{2}$, Eqs. (15) and (16), are:
As can be seen, the determinants of Jones matrices Eqs. (21) and (22) are in general case neither equal to zero nor to unity. For the Jones matrices, this implies the presence of dichroism [8], whereas, initial anisotropy of studied medium given by Eqs. (1) and (5) is purely circular birefringence and $\operatorname{det}\left(\mathbf{M}^{C P}\right)=1$.

Thus, the initial Mueller matrix Eq. (4) can be represented as a weighted sum of two deterministic Mueller matrices corresponding to the Jones matrices Eqs. (21) and (22) with weighted multipliers that are non-zero eigenvalues of Cloude's coherency matrix Eqs. (15) and (16).

Note that, as it results from Eqs. (15) and (16), in the case $\sigma_{h}=0$ we have only one non-zero Cloude's coherency matrix eigenvalue. This corresponds to the case of deterministic (non-depolarizing) class of objects. So, the appearance of one more non-zero eigenvalue is, hence, a result of the appearance of output light depolarization.

Generating the Mueller matrix Eq. (4) for various values of the inhomogeneity $\sigma_{h}$ and wavelength $\lambda$, obtaining for each Mueller matrix a corresponding Jones matrix $\mathbf{J}_{1}$ and decompose each Jones matrix $\mathbf{J}_{1}$ in the basis of the matrices of phase and amplitude anisotropy [8], we obtain the dependences of the values of circular dichroism $R$ and circular birefringence $\varphi$ on inhomogeneity $\sigma_{h}$ and wavelength $\lambda$. These dependences are presented in Figs 2 and 3, relatively.

$$
\begin{aligned}
& \mathbf{J}_{1}=\left(\begin{array}{cc}
\frac{\Phi_{12}+\Phi_{21}+\sqrt{\left(\Phi_{11}-\Phi_{22}\right)^{2}+4 \Phi_{12} \Phi_{21}}}{\Phi_{11}-\Phi_{22}-\Phi_{12}+\Phi_{21}} & -i \\
i & \frac{\Phi_{12}+\Phi_{21}+\sqrt{\left(\Phi_{11}-\Phi_{22}\right)^{2}+4 \Phi_{12} \Phi_{21}}}{\Phi_{11}-\Phi_{22}-\Phi_{12}+\Phi_{21}}
\end{array}\right) . \\
& \mathbf{J}_{2}=\left(\begin{array}{cc}
\frac{\Phi_{12}+\Phi_{21}-\sqrt{\left(\Phi_{11}-\Phi_{22}\right)^{2}+4 \Phi_{12} \Phi_{21}}}{\Phi_{11}-\Phi_{22}-\Phi_{12}+\Phi_{21}} & -i \\
i & \frac{\Phi_{12}+\Phi_{21}-\sqrt{\left(\Phi_{11}-\Phi_{22}\right)^{2}+4 \Phi_{12} \Phi_{21}}}{\Phi_{11}-\Phi_{22}-\Phi_{12}+\Phi_{21}}
\end{array}\right) .
\end{aligned}
$$

(C) 2009, V. Lashkaryov Institute of Semiconductor Physics, National Academy of Sciences of Ukraine 


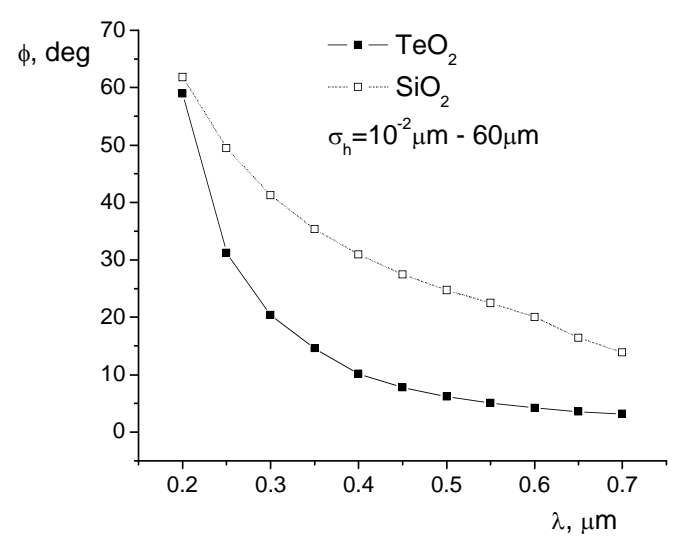

Fig. 3. Dependences of the values of circular birefringence $\varphi$ on inhomogeneity $\sigma_{h}$ and wavelength $\lambda$.

It can be seen that the values of circular birefringence $\varphi$ do not depend on inhomogeneity $\sigma_{h}$, and its behavior versus wavelength $\lambda$ is completely determined by the values of refractive indexes and their variation with changing $\lambda$.
The fact that, with the exception of $R$ and $\varphi$, all other types of anisotropy are absent enables to built the analytical expressions for the values of circular dichroism $R$ and circular birefringence $\varphi$. For that, we use the so-called second Jones' equivalence theorem [9], according to which the Jones matrix of the medium characterized only by circular dichroism and circular birefringence, $\mathbf{J}^{C B D}$, can be obtained in the following way:

$$
\mathbf{J}^{C B D}=\mathbf{J}^{C B} \mathbf{J}^{C D}=\left(\begin{array}{cc}
\cos (\varphi)+i R \sin (\varphi) & -i R \cos (\varphi)+\sin (\varphi) \\
i R \cos (\varphi)-\sin (\varphi) & \cos (\varphi)+i R \sin (\varphi)
\end{array}\right) .
$$

Introducing evident representation of the Jones matrix associated with the maximum eigenvalue Eq. (21) in the form:

$$
\mathbf{J}^{C B D}=\left(\begin{array}{cc}
j_{11} & -i \\
i & j_{11}
\end{array}\right),
$$

and making element wise comparison of the matrices Eqs. (23) and (24), for values $R$ and $\varphi$ we get

$$
\begin{aligned}
& R=\frac{1+\operatorname{Im}^{2}\left(j_{11}\right)+\operatorname{Re}^{2}\left(j_{11}\right)-\sqrt{\operatorname{Im}^{4}\left(j_{11}\right)+2 \operatorname{Im}^{2}\left(j_{11}\right)\left(\operatorname{Re}^{2}\left(j_{11}\right)-1\right)+\left(\operatorname{Re}^{2}\left(j_{11}\right)+1\right)^{2}}}{2 \operatorname{Im}\left(j_{11}\right)}, \\
& \varphi=\arccos \left(\sqrt{\frac{1+\operatorname{Im}^{4}\left(j_{11}\right)+2 \operatorname{Re}^{2}\left(j_{11}\right)+\operatorname{Re}^{4}\left(j_{11}\right)+t\left(1-\operatorname{Re}^{2}\left(j_{11}\right)-\operatorname{Im}^{2}\left(j_{11}\right)\right)-2 \operatorname{Im}^{2}\left(j_{11}\right)\left(1-\operatorname{Re}^{2}\left(j_{11}\right)\right)}{2 t^{2}}}\right),
\end{aligned}
$$

where $t=\sqrt{\operatorname{Im}^{4}\left(j_{11}\right)+2 \operatorname{Im}^{2}\left(j_{11}\right)\left(\operatorname{Re}^{2}\left(j_{11}\right)-1\right)+\left(\operatorname{Re}^{2}\left(j_{11}\right)+1\right)^{2}}$.

\section{Conclusion}

In summary, we have studied light scattering by inhomogeneous circular birefringent media in single scattering case. We derive the Mueller matrix model for this class of media and show that the resulting Mueller matrix for given observation angle implies the presence of circular dichroism, whereas, initial anisotropy of studied medium is purely circular birefringence. We show that for this medium the minimum value of polarization degree $p_{\min }^{\text {out }}$ is determined only by the values of refractive indexes at a given wavelength. Thus, the value $p_{\text {min }}^{\text {out }}$ cannot be equal to zero.

\section{References}

1. S.N. Savenkov, V.I. Grygoruk, R.S. Muttiah, K.E. Yushtin, Ye. Oberemok, V.V. Yakubchak, Effective dichroism in forward scattering by inhomogeneous birefringent medium JQSRT 110, p. 30-42 (2009).

2. E. Collett, Polarized Light: Fundamentals and Applications. Marcel Decker, New York, 1993, p. 360.
3. M.I. Mishchenko, L.D. Travis, A.A. Lacis, Scattering, Absorption, and Emission of Light by Small Particles. Cambridge, Cambridge University Press, 2002, p. 449.

4. Ch. Brosseau, Fundamentals of Polarized Light. A Statistical Optics Approach. New York, NorthHolland Publishing Company, 1998, p. 406.

5. S.R. Cloude, Group theory and polarization algebra // Optik (Stuttgart) 7, p. 26-36 (1986).

6. S.R. Cloude, E. Pottier, Concept of polarization entropy in optical scattering // Opt. Eng. 34, p. 1599-1610 (1995).

7. R. M. A. Azzam, N. M. Bashara, Ellipsometry and Polarized Light. North-Holland, Amsterdam, 1987, p. 356.

8. S.N. Savenkov, V.V. Marienko, E.A. Oberemok, O.I. Sydoruk, Generalized matrix equivalence theorem for polarization theory // Phys. Rev. E 74 056607 (2006).

9. H. Hurwitz, R. C. Jones, A new calculus for the treatment of optical systems II: Proof of three general equivalent theorems // Journ. Opt. Soc. Amer., 31, p. 493-499 (1941). 